\section{OPEN ACCESS}

Edited by:

Joaquim Ferreira,

Instituto de Medicina Molecular (IMM),

Portugal

Reviewed by:

Pedro Ribeiro,

Universidade Federal do Rio de

Janeiro, Brazil

Matteo Bologna,

Sapienza Università di Roma, Italy

*Correspondence:

Esther M. J. Bekkers

esther.bekkers@kuleuven.be

Specialty section:

This article was submitted to

Movement Disorders,

a section of the journal

Frontiers in Neurology

Received: 29 March 2018

Accepted: 18 June 2018

Published: 17 July 2018

Citation:

Bekkers EMJ, Van Rossom S, Heremans E, Dockx K, Devan S, Verschueren SMP and Nieuwboer $A$

(2018) Adaptations to Postural

Perturbations in Patients With

Freezing of Gait. Front. Neurol. 9:540.

doi: 10.3389/fneur.2018.00540

\title{
Adaptations to Postural Perturbations in Patients With Freezing of Gait
}

\section{Esther M. J. Bekkers ${ }^{1 *}$, Sam Van Rossom ${ }^{2}$, Elke Heremans ${ }^{1}$, Kim Dockx ${ }^{1}$, Surendar Devan ${ }^{1}$, Sabine M. P. Verschueren ${ }^{3}$ and Alice Nieuwboer ${ }^{1}$}

${ }^{1}$ Neuromotor Rehabilitation Research Group, Department of Rehabilitation Sciences, KU Leuven, Leuven, Belgium, ${ }^{2}$ Human Movement Biomechanics Research Group, Department of Movement Sciences, KU Leuven, Leuven, Belgium,

${ }^{3}$ Musculoskeletal Rehabilitation Research Group, Department of Rehabilitation Sciences, KU Leuven, Leuven, Belgium

Introduction: Freezing of gait (FOG) is a powerful determinant of falls in Parkinson's disease (PD). Automatic postural reactions serve as a protective strategy to prevent falling after perturbations. However, differences in automatic postural reactions between patients with and without FOG in response to perturbation are at present unclear. Therefore, the present study aimed to compare the response patterns and neuromuscular control between PD patients with and without FOG and healthy controls (HCs) after postural perturbations.

Methods: 28 PD patients (15 FOG+, 13 FOG-) and 22 HCs were included. Participants stood on a moveable platform while random perturbations were imposed. The first anterior platform translation was retained for analysis. Center of pressure (CoP) and center of mass (CoM) trajectories and trunk, knee and ankle angles were compared between the three groups using the Statistical Parametric Mapping technique, allowing to capture changes in time. In addition, muscle activation of lower leg muscles was measured using EMG.

Results: At baseline, FOG+ stood with more trunk flexion than HCs ( $p=0.005)$, a result not found in $\mathrm{FOG}-$. Following a perturbation, $\mathrm{FOG}+$ reacted with increased trunk extension $(p=0.004)$ in comparison to $\mathrm{HCs}$, a pattern not observed in FOG-. The CoM showed greater backward displacement in FOG- and FOG + $p=0.008$, $p=0.027)$. Both FOG+ and FOG - showed increased co-activation of agonist and antagonist muscles compared to HCs $(p=0.010)$, with no differences between FOG+ and FOG-.

Conclusions: Automatic postural reactions after a sudden perturbation are similar between PD subgroups with and without FOG but different from HCs. Reactive postural control, largely regulated by brain stem centers, seems to be modulated by different mechanisms than those governing freezing of gait. Greater differences in initial stance position, enhanced by joint stiffening, could however underlie maladaptive postural responses and increase susceptibility for balance loss in FOG+ compared to FOG-.

Keywords: postural control, freezing of gait, Parkinson's disease, perturbations, falls, reactive postural control 


\section{INTRODUCTION}

When a stable posture is suddenly perturbed, the body must react and quickly adjust to recover balance. These reactive postural adjustments are considered to be automatic responses, since the activation onset of muscle contraction is shorter than voluntary reaction times (1). As a consequence of neuronal loss in the basal ganglia, patients with Parkinson's disease (PD) present with impaired motor automaticity particularly during voluntary repetitive sequential movements (2). However, continuous automatic motor control (allowing voluntary motor activity without conscious thought), may not necessarily cover automatic instantaneous postural responses as well. So far, studies on postural reactions to external perturbations showed delayed, inflexible and inefficient balance correcting responses in PD (3-6). The release of automatic postural responses involves brainstem structures, including the mesencephalic locomotor region, as well as basal ganglia input, particularly from the striatum (7-9). These regions are also known to be involved in freezing of gait (FOG) (10). People with FOG (FOG+) have larger impairments in central drive and movement automaticity than their non-freezing counterparts (11). The higher fall risk in this subgroup may possibly be explained by greater deficits in automatic postural control as well. In addition, larger impairments in reactive postural control may underlie the finding that when using clinical balance scales more severe balance deficiencies are apparent in FOG+ $(12,13)$.

The current state of the art on comparisons of responses to sudden balance perturbations in FOG+ vs. FOG- revealed that FOG+ had smaller balance corrective steps compared to FOG- (14). Hence, FOG+ seemed to either underestimate the size of the corrective step response needed to recover balance or tended to release responses with reduced gain. In contrast, two other studies investigating freezing-related balance control after sudden perturbations found no differences in Center of Mass (CoM) excursions or protective stepping behavior between groups with and without FOG $(8,15)$. However, FOG+ were less able to improve protective postural responses after repetitive perturbations $(14,15)$. Although the amplitudes of reactive postural responses and the quality of the first balance correcting step did not differ during forward platform translations, an attenuated response was found when providing a starting stimulus to accelerate responses in FOG+ (8). FOG+ showed delayed onset latencies and reduced acceleration responses of the tibialis anterior and rectus femoris compared to FOG- and healthy controls (8).

These previous studies mainly investigated reactive stepping, induced by compensatory behavior in response to perturbation. Until now, no study has compared the neuromuscular control of the lower limbs during perturbation between subgroups in PD, although earlier work showed attenuated agonist and increased

\footnotetext{
Abbreviations: CCI, Co-Contraction Index; CoP, Center of pressure; FOG, Freezing of Gait; FOG+, Patients with FOG; FOG-, Patients without FOG; GM, Gastrocnemius Medialis; HCs, Healthy controls; PD, Parkinson's disease; SPM, Statistical Parametric Mapping; TA, Tibialis Anterior; (X)CoM, (Extrapolated) Center of mass.
}

antagonist activation and higher background activity in lower legs muscles in PD in comparison to controls $(5,6)$. FOG+ showed more flexed posture and altered electromyography (EMG)-patterns in the Tibialis Anterior (TA) and Medial Gastrocnemius (GM) during gait, especially in the gait cycles preceding freezing episodes $(16,17)$.

Unlike previous work, the current study aimed to unravel postural response differences between patients with and without FOG and healthy elderly, while a stable posture is maintained during perturbations. Compromised adaptive behavior in FOG was previously demonstrated during splitbelt treadmill perturbations during walking and suggested to be related to a reduced perception of locomotor asymmetry $(18,19)$. Adaptive behavior is time-varying and cannot fully be described by extracting one summary scalar at particular time points or regions of the motion trajectory. Therefore, in the current study we focused on postural adaptations during backward responses, which is the most common type of disequilibrium in aging and $\mathrm{PD}(5,6,20)$. We also chose to analyze our force plate and kinematic data using statistical parametric mapping (SPM) to capture extended trajectories of adaptive movement. We hypothesized that due to reduced automatic reactions, FOG+ would show greater instability in response to external perturbations compared to FOG- and HCs. In addition, EMG signals during postural reactions were compared between groups to unravel potential underlying abnormalities of neuromuscular control.

\section{MATERIALS AND METHODS}

Twenty-nine Parkinson's disease and 22 healthy elderly adults (HCs) were included in the study. PD patients were categorized as FOG $+(n=16)$ if they scored 1 on item 1 of the new Freezing of Gait Questionnaire (NFOG-Q) or FOG- $(n=13)$ if they scored 0 on this item. All participants met the inclusion criteria of a Mini-Mental State Examination (MMSE) $>23$ and were able to stand upright for at least $15 \mathrm{~min}$. In addition, inclusion criteria for the patient groups were: (i) diagnosis of PD based on the United Kingdom Parkinson's Disease Brain Bank Criteria (21), (ii) Hoehn and Yahr (H\&Y) stage II or III (in ON-medication), and (iii) being on stable medication for the last month. Exclusion criteria comprised having a neurological disorder other than $\mathrm{PD}$, vestibular disorders, musculoskeletal disorders or any other disease that could interfere with the experimental task. Patients were tested during the $\mathrm{ON}$-medication state. The study was approved by the local ethical committee of the University Hospitals Leuven (s54665) and all participants provided written informed consent.

\section{Test Protocol \\ Clinical Assessment}

Clinical assessments included demographic data, Montreal Cognitive Assessment (MoCA) and MMSE. The Mini Balance Evaluation System Test (Mini-BEST) was used to clinically assess postural control, including subdomain scoring (22). Disease severity in PD patients was determined by the Movement 
Disorder Society Unified Parkinson's Disease Rating Scale part III (MDS-UPDRS-III).

\section{Data Recording}

The Computer Assisted Rehabilitation Environment (CAREN) movable platform operated with D-Flow software (Motek Forcelink, Amsterdam, The Netherlands) was used to randomly perturb posture to elicit postural responses. Three-dimensional marker trajectories were captured using 7 infrared cameras (Vicon, Oxford Metrics, UK, $100 \mathrm{~Hz}$ ). Forty-five spherical reflective markers were placed on specific anatomic landmarks, using an adapted version of the Liverpool John Moores University model (23). Simultaneously, ground reaction forces were recorded using two force plates integrated in the CAREN platform (AMTI, Watertown, USA, 1,500 Hz). Lastly, surface EMG signals (Aurion, Zero-wire, IT, 1,500 Hz) of the tibialis anterior (TA) and gastrocnemius medialis (GM) were recorded during balance reactions $(\mathrm{FOG}+=15, \mathrm{FOG}-=12, \mathrm{HCs}=19$ ). EMG data could not be sampled in all patients $(N=5)$ due to technical problems or due to not being able to lengthen the study in some cases. Electrodes were placed according to the SENIAM-guidelines.

Participants were asked to stand on the platform, looking straight ahead with arms crossed over the chest. Feet were slightly externally rotated at a standardized width of $15 \mathrm{~cm}$ between medial malleoli. Participants were instructed to keep their balance without moving their arms or taking a step as the platform moved, unless falling was imminent. All subjects wore a safety harness to prevent falling. The platform was accelerated by $1.5 \mathrm{~m} / \mathrm{s}^{2}$, whereby the translation amplitude was standardized using the height of the participants as a scaling factor. Participants' posture was perturbed by random translations in four directions. The first anterior platform translations, imposing a backward balance perturbation, were retained for further analysis (24), as it was found earlier that this would provide the most revealing information on reactive postural control precluding learning effects.

\section{Data Analysis}

A customized bodybuilder kinematic model was used to determine 3D joint angles from the measured marker trajectories (Vicon BodyBuilder, Oxford Metrics). Ankle and knee angles were calculated as relative joint angles, expressing the relative angle between the two adjacent segments whereas the trunk angle was calculated as the angle relative to the vertical axis of the global reference frame. Ground reaction forces were first filtered using a 4th order low-pass Butterworth filter with a cut-off frequency of $10 \mathrm{~Hz}$ before calculating the combined Center of Pressure (CoP). Marker positions on the different anatomical landmarks were used to calculate the whole-body CoM, accounting for the relative mass of each individual segment. Patterns for SPM analyses were corrected to the initial position to account for different starting position. Peak values were extracted to indicate absolute position in space. The CoM and CoP were additionally corrected for platform movement. The extrapolated $\mathrm{CoM}$ (XCoM) was then calculated as measure of dynamic stability, according to the formula $X C o M=p+\frac{v}{\omega_{0}}$, where $\omega_{0}=\sqrt{\text { gravity/vertical CoM position }}, p$ is the CoM position and $v$ the initial CoM velocity (25). Raw EMG signals were first bandpass filtered using a 4th order Butterworth filter between 20 and $500 \mathrm{~Hz}$, subsequently the signal was full-wave rectified. Lastly, a linear envelope of the signal was determined by applying a 4th order Butterworth low-pass filter with $40 \mathrm{~Hz}$ cut-off.

In analogy to previous studies, EMG signals measured from the TA and GM were quantified. Raw EMG signals were corrected by subtracting background activity, averaged of a period from 200 to $50 \mathrm{~ms}$ before perturbation onset (24, 26). EMG records were first normalized to maximum muscle activity over anterior and posterior perturbation conditions per subject. Magnitude of the corrected EMG signal was calculated by averaging over a time window $80-450 \mathrm{~ms}$ after perturbation onset (27). Muscle co-contraction index (CCI) of the GM-TA was calculated to determine antagonist/agonist activity ratio by applying the following calculation (28): $\mathrm{CCI}=2^{*}\left(\mathrm{EMG}_{\text {ant }} / \mathrm{EMG}_{\mathrm{ag}}+\mathrm{EMG}_{\mathrm{ant}}\right)^{*} 100$. For the trials in which a protective step was necessary to maintain balance, step length and onset latency were calculated. Step length was calculated based on the heel marker position of the stepping leg. Step onset was determined as the time between perturbation onset and foot lift-off, identified from a vertical GRF lower than $10 \mathrm{~N}$. Stable, in-place responses were used for further posturography and EMG analysis. All variables were analyzed from $200 \mathrm{~ms}$ prior to $1,000 \mathrm{~ms}$ after perturbation onset.

\section{Statistical Analysis}

Statistical analysis was performed using IBM SPSS software (version 22). Demographic characteristics were compared between groups using ANOVAs, Kruskal-Wallis ANOVAs, independent sample $t$-test or Mann-Whitney $U$-tests, depending on the distribution of the variables. Categorical data were analyzed using Chi-square statistics. Abnormally distributed postural data were compared between groups using nonparametric Kruskal-Wallis ANOVA's and Chi-square for frequency data. Significant results were further investigated using a Mann-Whitney $U$-tests for post-hoc comparisons. Pattern analyses were performed using spm1d (non-parametric hypothesis testing) (v0.3) (www.spm1d.org). EMG measures were normally distributed and compared between groups using ANCOVAs. To account for differences in group characteristics, age was added as covariate. The critical threshold was set at $p<0.05$, but Bonferroni-corrected for the post-hoc analysis.

\section{RESULTS}

\section{Demographics}

Participants' characteristics are presented in Table 1. FOG+ and FOG- were well-matched for all demographic and diseaserelated parameters $(p>0.05)$. HCs however, were significantly older compared to FOG $+(p=0.001)$ and FOG- $(p=0.012)$. Cognitive measures were similar between groups. Both FOG+ and FOG - showed more falls compared to HCs $(p<0.001$; $p=0.031$ ), but no subgroup differences were found. Scores on the Mini-BEST also differed significantly between HCs and PD 
TABLE 1 | Participants' demographics.

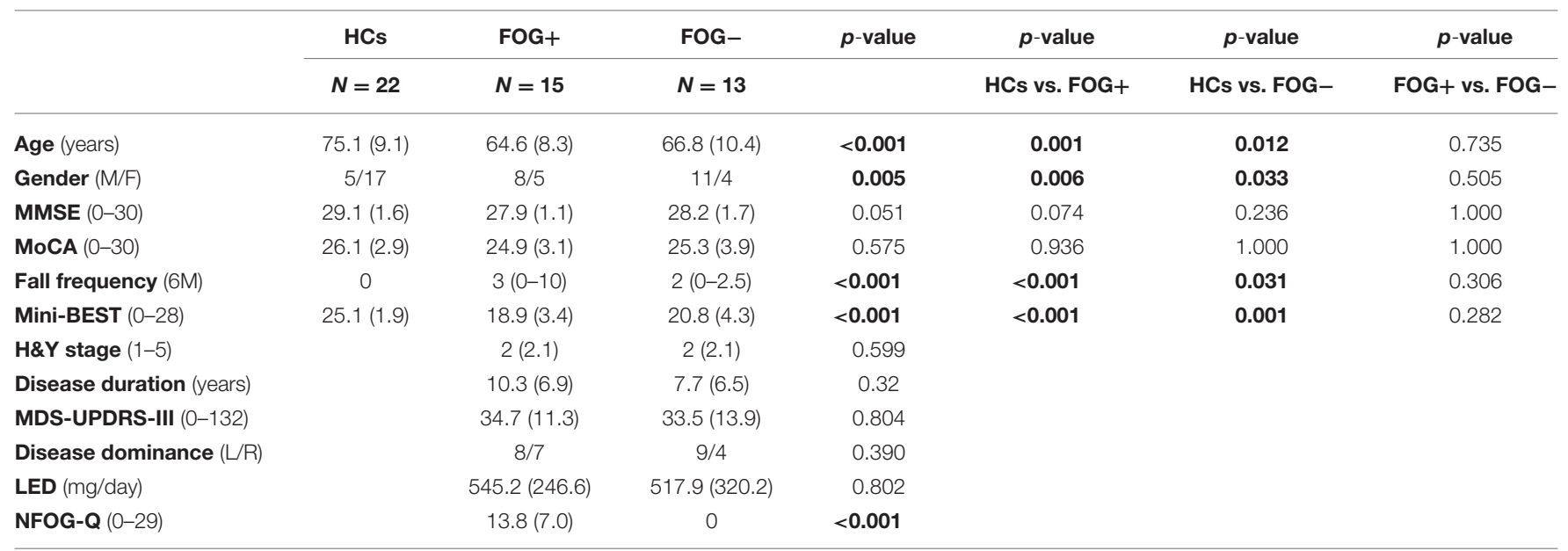

Means ( $\pm S D$ ) or median (interquartile range 25-75\%) are reported. P-values below the significance level of 0.05 are indicated in bold.

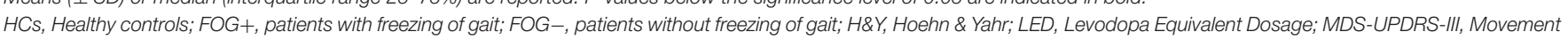

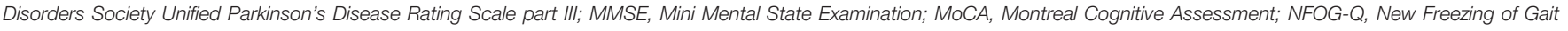
Questionnaire.

subgroups $(p<0.001)$, but no dissimilarities were found between FOG + and FOG - . Analysis of the subcomponents of the MiniBEST revealed that the differences in the PD subgroups vs. HCs were most pronounced on reactive $(p<0.001)$ and dynamic $(p<0.001)$ postural control, with no apparent differences between FOG + and FOG-. One patient with FOG was unable to complete the platform test and was excluded from data analysis.

\section{Stepping Responses}

Stepping responses did not differ between groups. Percentage protective stepping responses were higher in both FOG+ $(33.3 \%)$ and FOG - (30.8\%) compared to HCs (22.7\%), but did not differ significantly between these cohorts $\left[\mathrm{X}_{(2)}=0.564, p=0.754\right]$. Average step lengths were smaller in both PD groups, but were not significantly different from $\mathrm{HCs}$ (FOG+: $25.27 \pm 17.12 \mathrm{~cm}$; FOG-: $24.89 \pm 17.36 \mathrm{~cm}$, HCs: $36.41 \pm 18.26 \mathrm{~cm}$; $p=0.589)$. Similarly, no differences were found in step latency between groups (FOG+: $385 \pm 30$ ms; FOG-: $314 \pm 39$ ms, HCs: $358 \pm$ $50 \mathrm{~ms} ; p=0.141)$.

\section{Kinematics and Kinetics During Non-stepping Postural Response Initial Stance}

During the time interval before platform translation onset (0$200 \mathrm{~ms}$ ), significant differences between FOG+, FOG-, and HCs were found for both trunk $(p=0.01)$ and knee position $(p=0.01)$. FOG + had a more stooped posture compared to HCs (trunk flexion: $19.61 \pm 11.82$ vs. $9.04 \pm 4.69, p=0.005$ ), unlike FOG-. No differences were revealed between FOG + and FOG-. Both PD subgroups had also more knee flexion (FOG+17.13 \pm 10.23 ; FOG $-17.31 \pm 9.28)$ compared to HCs $(7.82 \pm 6.87)$ $(p=0.005 ; p=0.005)$.

\section{Postural Responses}

SPM

Kinematic data revealed a significant difference in trunk movement following a backward balance perturbation, this despite correcting for increased trunk flexion at baseline (significant between 267-368 msec following perturbation, $p=0.015$ ) (Figure 1A). FOG + responded with increased trunk extension compared to HCs between 184-554 ms after perturbation onset $(p=0.004)$ (Figure 1D), a pattern not observed in FOG- (Figure 1E). No differences were shown between FOG- and FOG+ (Figure 1F), probably due to higher variability in the FOG group. No significant differences between groups regarding knee and ankle angles were found (Figures 1B,C).

Furthermore, XCoM responses differed significantly between groups immediately after perturbation onset (137-239 ms, $p=0.025$ ) (Figure 2). Group comparisons revealed increased backward XCoM responses for FOG-compared to HCs at time interval 76-264 ms ( $p=0.008)$ (Figure 2D). No differences were found between HCs and FOG+, although FOG+ tended to have greater backward CoM displacement during postural reactions especially at a later time zone (Figure 2C). In FOG+ more variable responses can be noted. FOG + and FOG- did not differ regarding XCoM displacement, nor were group differences found for CoP.

Pooling the results of PD subgroups for the SPM analyses showed increased trunk extension between 115-492 ms $(p=0.004)$ and more backwards directed XCoM between $210-264 \mathrm{~ms}(p=0.036)$ in PD vs. HCs (Supplementary Figure 1).

\section{Peak values}

Table 2 displays overall peak value differences between groups. Similar to the SPM analysis, greater trunk displacement was shown in FOG+ compared to HCs $(p=0.025)$. Absolute peak extension position was however not different between groups 

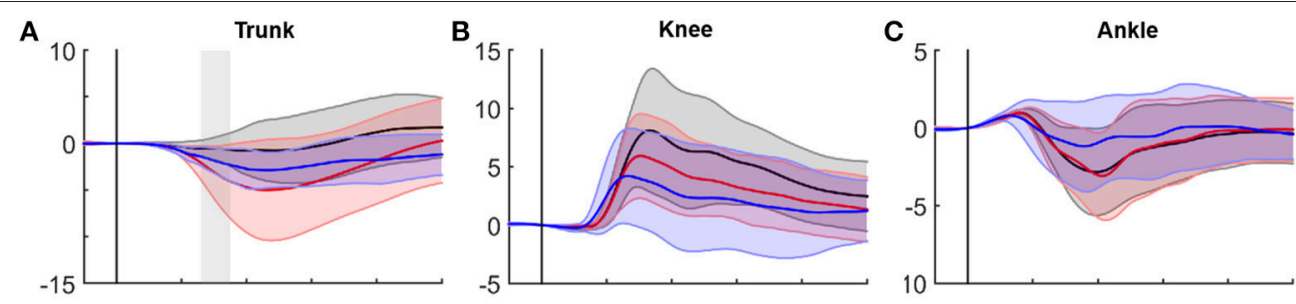

Trunk: Post-hoc comparisons
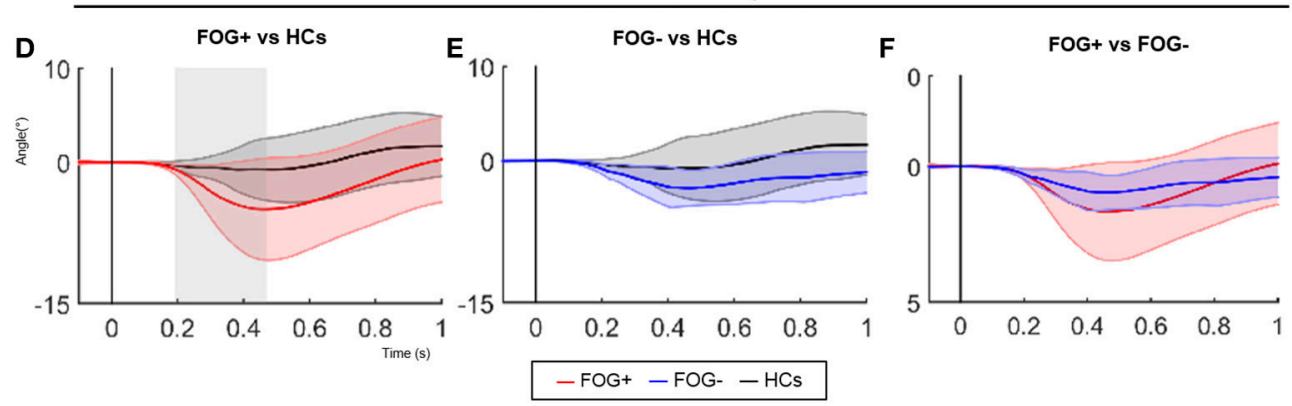

FIGURE 1 | SPM analysis of joint angle responses following a posterior perturbation. Average $( \pm S D)$ of angular displacement $\left({ }^{\circ} \Delta\right)$ following a posterior perturbation in the three groups is shown in figure 1 (upper panels). Lower panels show post-hoc comparisons of the trunk pattern between groups. Gray zones indicate the time zones where groups significantly differ.

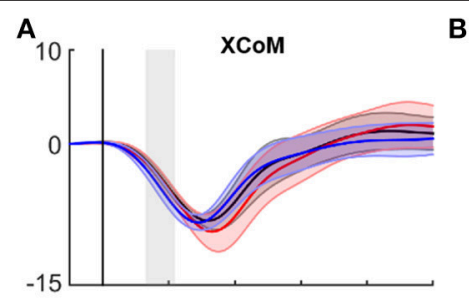

B CoP
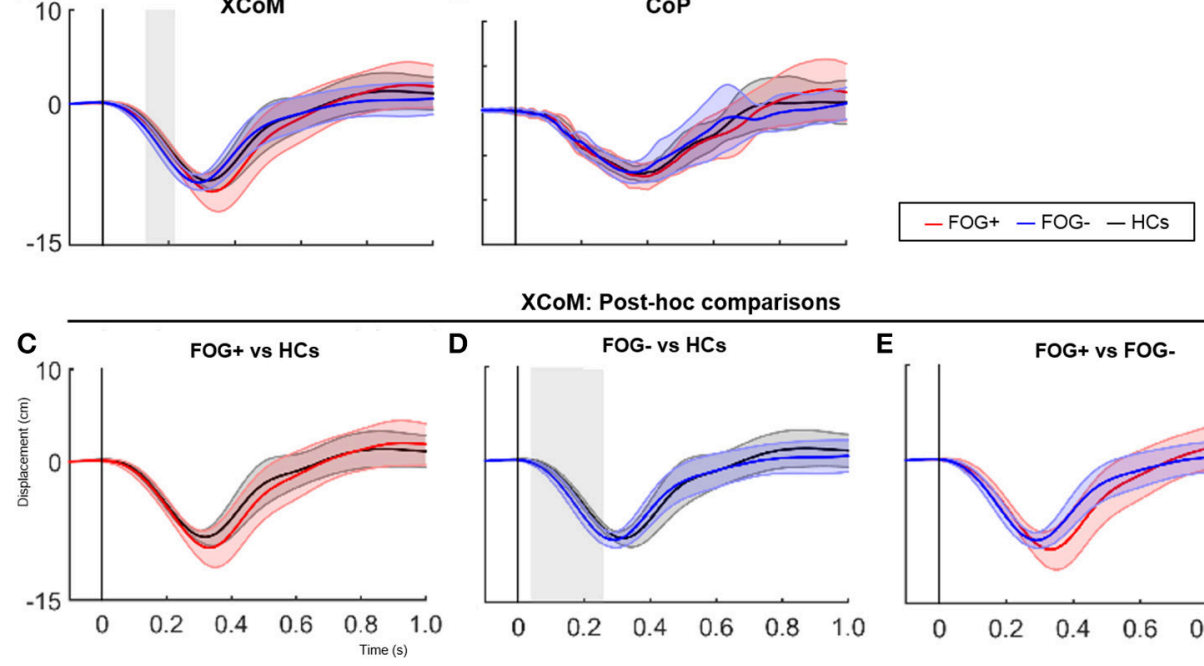

XCoM: Post-hoc comparisons
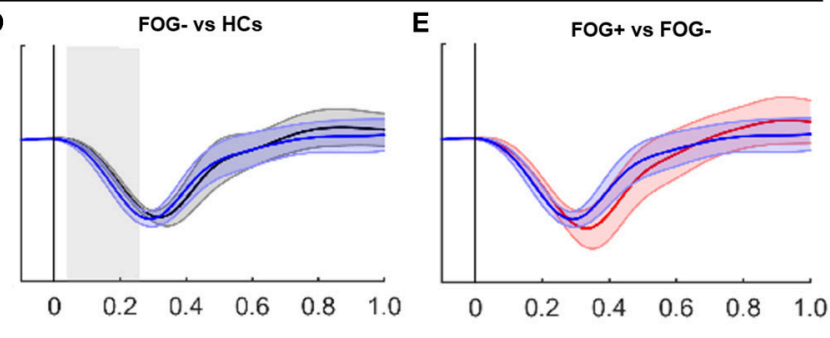

FIGURE 2 | SPM analysis of XCoM and CoP responses following a posterior perturbation. Average ( \pm SD) of XCoM and CoP displacement (cm) following a posterior perturbation in the three groups is shown in figure 2 (upper panels). Lower graphs show post-hoc comparisons between groups for XCoM. Gray zones indicate the time zones where groups significantly differ from each other.

$(p=0.052)$, indicating that FOG + moved not beyond the peak extension position of HCs. A similar pattern was found for knee angles, showing significant greater maximal knee flexion in FOG + compared to HCs $(p=0.031)$, but the range of motion did not differ between groups. No differences in ankle joint angles and no differences compared to FOG- were found. Furthermore, larger XCoM displacement was seen in FOG+ compared to HCs $(p=0.027)$, but not in FOG-. XCoM time to peak and $\mathrm{CoP}$ parameters did not show any differences in postural response.

Pooled results showed significantly increased knee flexion in PD compared to HCs at maximal knee position $(p=0.007)$, but the total knee range of motion was smaller in $\mathrm{PD}(p=0.031)$ (Supplementary Table 1). No other differences in angular responses were found. Also, $\mathrm{CoP}$ and $\mathrm{XCoM}$ parameters showed no differences between groups. 
TABLE 2 | Peak values of kinematic and kinetic data.

\begin{tabular}{|c|c|c|c|c|c|c|c|c|}
\hline & & HCs & FOG+ & FOG- & $p$-value & HCs vs. FOG+ & HCs vs. FOG- & FOG + vs. FOG- \\
\hline \multirow[t]{3}{*}{ Peak $\left({ }^{\circ}\right)$} & Trunk & $6.88(4.15)$ & $14.21(9.64)$ & 8.05 (10.14) & 0.052 & 0.055 & 1.000 & 0.276 \\
\hline & Knee & $17.24(4.61)$ & $23.78(7.87)$ & $22.33(7.23)$ & 0.027 & 0.031 & 0.164 & 0.875 \\
\hline & Ankle & $7.01(4.95)$ & 8.05 (4.63) & $9.47(5.57)$ & 0.518 & 1.000 & 0.774 & 1.000 \\
\hline \multirow[t]{3}{*}{$\operatorname{ROM}\left({ }^{\circ} \Delta\right)$} & Trunk & $4.12(2.11)$ & $7.35(7.5)$ & $4.01(1.58)$ & 0.017 & 0.025 & 1.000 & 0.064 \\
\hline & Knee & $9.97(4.80)$ & $7.18(3.78)$ & $6.30(3.54)$ & 0.093 & 0.302 & 0.160 & 1.000 \\
\hline & Ankle & $5.14(2.31)$ & $5.18(2.61)$ & $3.57(1.82)$ & 0.244 & 1.000 & 0.365 & 0.427 \\
\hline XCoM Peak (cm) & & $8.57(0.10)$ & $9.67(1.8)$ & $8.52(0.70)$ & 0.023 & 0.027 & 1.000 & 0.125 \\
\hline XCoM Time to Peak (s) & & $0.34(0.03)$ & $0.35(0.03)$ & $0.33(0.02)$ & 0.134 & 0.199 & 1.000 & 0.320 \\
\hline CoP Peak (cm) & & $7.46(9.13)$ & $7.68(1.26)$ & 7.39 (1.19) & 0.668 & 1.000 & 1.000 & 1.000 \\
\hline CoP Time to Peak (s) & & $0.38(0.13)$ & $0.36(0.15)$ & $0.34(0.11)$ & 0.827 & 1.000 & 1.000 & 1.000 \\
\hline
\end{tabular}

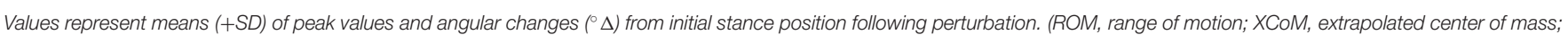
CoP, center of pressure).

\section{Muscle Responses}

Electromyography (EMG) responses are shown in Figure 3A. The magnitudes of the left and right signals did not differ between groups and were therefore pooled to increase power. Magnitudes of TA activation showed no differences between groups $(p=0.084)$. The EMG activity of GM muscles showed group differences $(p=0.039)$, with larger magnitudes in FOGvs. HCs $(p=0.036)$, whereas FOG + did not differ from both groups.

Overall, both PD groups showed increased antagonist activity (GM) and reduced agonist activity (TA) in comparison to HCs. This was also illustrated by a significant increase in cocontraction (CCI) $(p=0.004)$ (Figure 3B). Compared to HCs, both FOG+ and FOG- showed increased co-contraction of lower leg muscles $(p=0.034, p=0.005)$. Examples of these different activation patterns of agonist and antagonist are shown in Figure 4. No differences were found between FOG+ and FOG-. Analysis of the pooled data of PD subgroups showed increased GM activity ( $p=0.012)$ compared to HCs, whereas no differences were found for TA. As a result, PD patients showed increased GM-TA co-contraction $(p=0.005)$ in response to a backward balance perturbation (Supplementary Figure 2).

\section{DISCUSSION}

This study aimed to compare postural adaptation patterns and neuromuscular control during backward balance responses in PD patients with vs. without FOG. Previous work using clinical balance assessments indicated that postural control is significantly worse in freezers and that this difference is most pronounced during reactive postural control in response to a leaning task (13). PD subgroups in the present study were wellmatched for clinical profiles, including clinical balance outcomes and fall rates. Detailed posturographic and electromyographic data showed differences between FOG+ and HCs, which were not present in FOG-. However, no specific freezing-related differences were found. Abnormal trunk response patterns in freezers were demonstrated, revealing a larger degree of trunk extension directly following a backward balance perturbation in

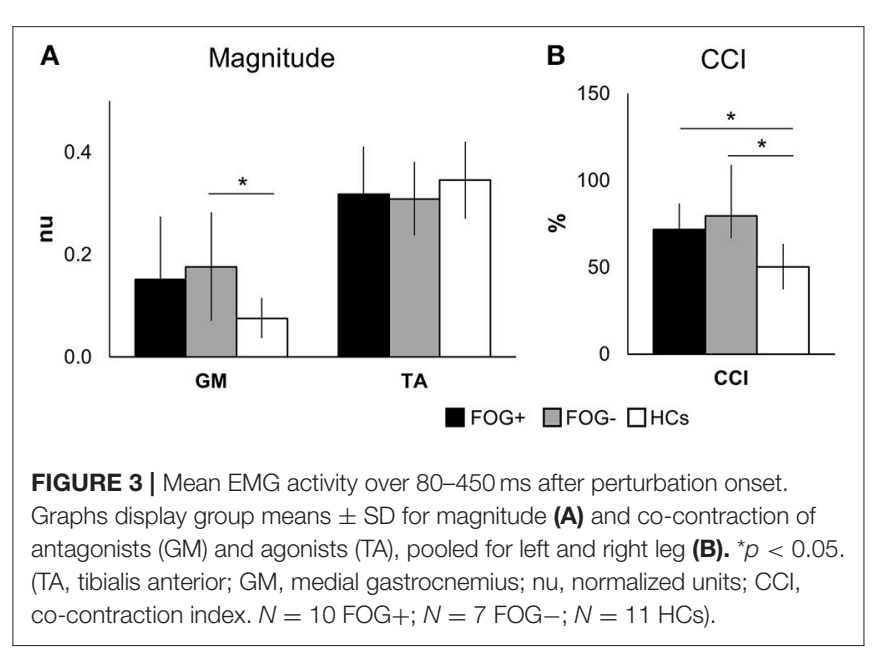

FOG+ compared to HCs. The XCoM results showed greater backward displacement in both FOG+ and FOG - than in HCs. Equally, EMG outcomes indicated increased co-activation of lower leg muscles, which were similar in both PD groups. Our results indicate that reactive postural control is largely comparable between subgroups with and without FOG and therefore our hypothesis was not confirmed. However, FOG+ have more pronounced abnormal reactive responses in the trunk when compared to HCs, and this when fall rate and balance capacity was equal in FOG + and FOG-.

Both FOG + and FOG-, who were able to counteract the balance perturbation with an in-place response, showed more retropulsive dyscontrol bringing subjects closer to their limits of stability, suggesting that PD patients were more unstable directly following the perturbation. Earlier research also showed increased backward instability in PD patients compared to HCs, irrespective of medication $(4,29)$ and a more backward shifted $\mathrm{CoP}$ position in FOG + compared to FOG - and HCs during normal upright stance (30). Together, these findings explain the greater tendency for backward falls in $\operatorname{PD}(5,20)$. The maximally extended position was however not different from 


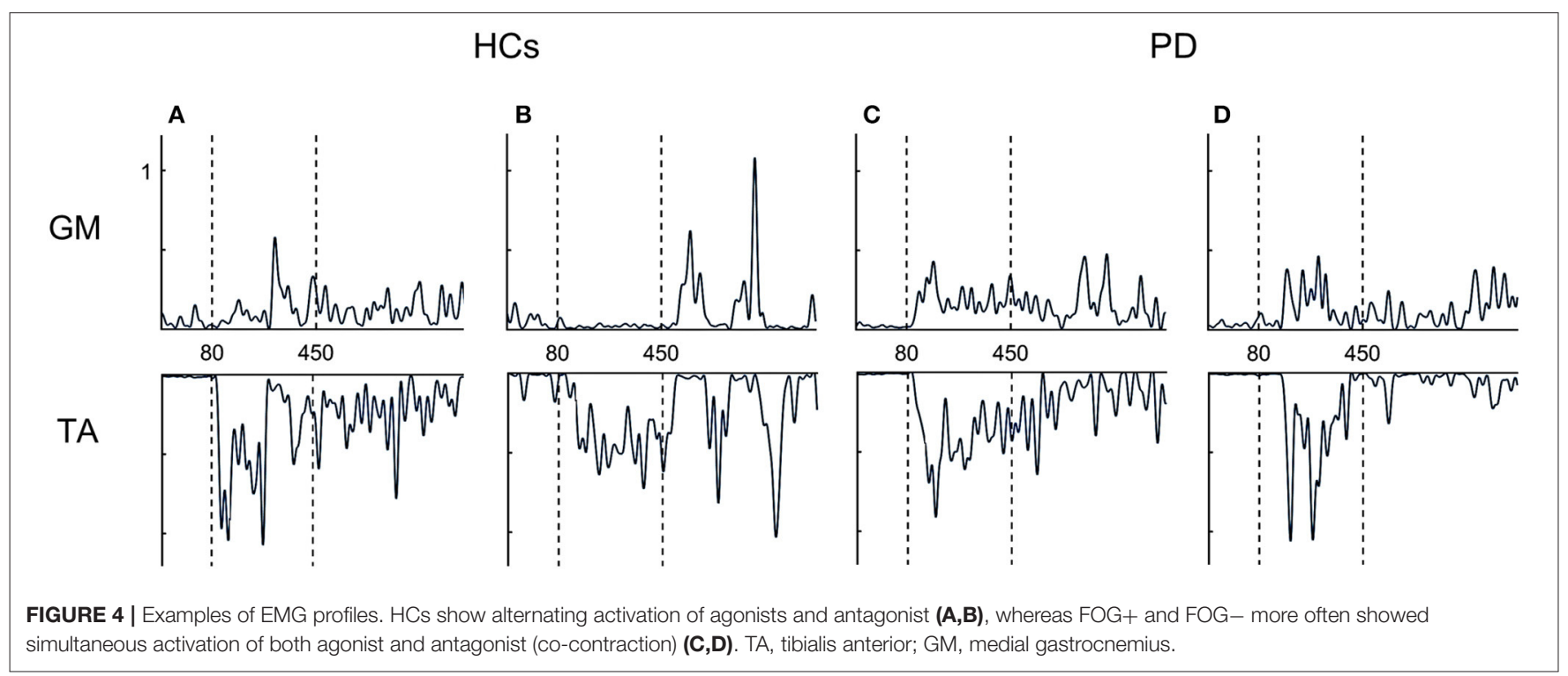

HCs. Backward instability may be amplified by the increase in stooped position during initial stance, which enables to generate greater backward momentum when extending the trunk. Our results showed that FOG + had an increased trunk and knee flexion, and reacted with exaggerated trunk extension following a perturbation. This could induce an even higher risk of backward falling in this subgroup. Earlier work suggested that stooping is a destabilizing factor, but does not solely explain postural instability in PD (31). As such, differences in initial joint position could trigger postural responses that increase susceptibility for balance loss. Whereas the XCoM was further backwards following the perturbation, as shown by both the SPM analysis and peak values in both FOG+ and FOG- compared to HCs, no differences in $\mathrm{CoP}$ were found. This can be explained by the fact that for stable responses, $\mathrm{CoP}$, and CoM measures have a certain ceiling effect as these only vary within the limits of stability.

Patients with FOG+ did not differ in performing a corrective step to maintain balance compared to FOG- or HCs. This result is in line with those of Nonnekes et al., reporting no differences in stepping responses (8), although smaller steps in FOG + compared to FOG- have also been reported (14). In addition, our findings support earlier research showing that switching to an alternative response strategy from feet-in-place trials to change-in-support, was similar between FOG+, FOG-, and HCs (14). This underscores that both patients with FOG+ and FOG- may not be more inflexible per se, but rather choose an inappropriate kinematic strategy to control their body. On the other hand, this study shows that the differences in kinematic responses between PD and HCs could be the result of inappropriate muscle coordination during a backward balance perturbation. At the muscular level, agonists should be activated in order to counteract for the backward loss of balance during a forward platform translation. Both FOG+ and FOG- showed increased antagonist activity of lower leg muscles (GM) in relation to reduced agonist activity (TA) compared to HCs. This led to increased co-activation of lower leg muscles in $\mathrm{PD}$, which probably contributes to increased limb stiffness and less postural flexibility (1). Indeed, our data showed smaller ankle responses and a significantly reduced knee range of motion in PD vs. HCs. This stiffening of lower limbs could have resulted in a compensatory exaggerated trunk movement in PD, whereas HCs where able to respond from their knees. Therefore, stiffening of lower legs is seemingly also an explanatory factor for the typical retropulsion difficulties in PD. This finding is further supported by the fact that previous studies already showed earlier activation onset, increased magnitude of antagonist muscles and active stiffening in PD vs. HCs following a balance perturbation $(5,6)$. Although not examined directly, results of the present study confirm these findings and further extend these by demonstrating that PD subgroups with and without FOG show the same exaggerated co-activation during postural perturbations.

The basal ganglia play an important role in the regulation of muscle tone and coordination, mediated via reciprocal projections to the brainstem and cerebral areas. As such, they constitute fundamental structures for postural control $(9,32$, 33). Whereas the basal ganglia-brainstem pathways contribute to automatic movement control and regulate muscle tone (32, 33), reactive postural responses are mainly mediated by brain stem centers per se, particularly the pontomedullary reticular formation ( $\mathrm{pmRF}$ ) and pedunculopontine nucleus $(\mathrm{PPN})(7,34)$. These notions come from studies investigating the StartReact phenomenon, in which responses are accelerated by triggering sensory stimuli. Hereby, it is assumed that some motor programs are subcortically stored in a prepared state and can be released quickly when triggered (7). The StartReact effect was shown to be attenuated in patients with FOG, but was intact in patients with postural instability (8). Additionally we showed that, in line with previous literature, automatic postural reactions are similar between PD subgroups. Reactive postural control therefore seems to affect different mechanisms than those governing FOG. However, this hypothesis remains speculative, was not 
directly assessed in the present study and requires further investigation.

The fact that slightly different results were found between groups on peak values vs. the pattern analyses, indicates that automatic postural reactions are also highly individualized. The individual nature of postural strategies was recently demonstrated in a study regarding anticipatory postural control, showing that anticipatory postural adjustments (APAs) prior to gait initiation differ between healthy people in general and that these differences are not related to aging (35), and possibly also not to disease. As such, postural differences may also be difficult to generalize to a population as a whole. Intriguingly, the variability in the FOG+ group was even more substantial. This highlights also the need for a more individualized approach in rehabilitation. For PD, exercise programs showed to improve kinematic measures and efficient muscle coordination in response to perturbations $(27,36)$. However future studies should confirm whether the effects for patients with FOG are similar as those for a general PD population. This is particularly of interest as FOG+ are known to have learning deficiencies that affect both motor and cognitive performance $(14,15,37)$. This could therefore result in slower adaptation of muscle coordination. As such, FOG-related deficits are important to take into account to optimize rehabilitation programs for freezers.

Although this study provided more insights into the contributing factors to postural instability in PD, several limitations need to be taken into account. As indicated above, in postural control data, there is large variability, which hampers parametric statistics as well as the correction for confounding variables. This also points to the fact that strategies to maintain stability are dependent on individual strategies and therefore kinematic measures are difficult to use as reliable 'predictors' of reactive postural control. Secondly, the elderly controls were significantly older than the PD patients, which may have resulted in an underestimation of our results. However, to counteract this effect, we added age as a covariate to our analyses. Furthermore, PD patients were measured while ON medication, which better reflects the real-life situation in which falls mainly occur, but can therefore not be generalized to the OFF state. In order to limit the confounding influence of medication, the PD subgroups with and without FOG were adequately matched. Moreover, dopaminergic medication may fail to improve postural impairment $(4,38,39)$. Others indicated that medication has a highly variable impact on postural control (40). Testing in both ON and OFF state could, however, provide a more complete picture of freezingrelated postural control, which would be valuable to assess in

\section{REFERENCES}

1. Rinalduzzi S, Trompetto C, Marinelli L, Alibardi A, Missori P, Fattapposta F, et al. Balance dysfunction in Parkinson's disease. Biomed Res Int. (2015) 2015:434683. doi: 10.1155/2015/434683

2. Wu T, Hallett M. A functional MRI study of automatic movements in patients with Parkinson's disease. Brain (2005) 128:2250-9. doi: 10.1093/brain/awh569

3. Horak FB, Nutt JG, Nashner LM. Postural inflexibility in parkinsonian subjects. J Neurol Sci. (1992) 111:46-58

4. Bloem BR, Beckley DJ, van Dijk JG, Zwinderman AH, Remler MP, Roos RA. Influence of dopaminergic medication on automatic postural responses and future studies. Further, we only measured EMG of the lower limbs. Based on our results, hip and trunk muscle responses may have provided additional information and could be of interest for further investigation. Lastly, the small sample size may have resulted in an underestimation of significant results and/or caused a lack of significant findings. EMG could not be sampled in all subjects and about $30 \%$ of our total participants reacted with a stepping response, not only limiting more detailed analysis of these patients' kinetic and kinematic measures but also could have biased the results. This should be taken into account for sample size calculations in future research.

To conclude, automatic postural reactions after a sudden perturbation are largely similar between subgroups of PD with and without FOG, but those with FOG have more abnormal responses compared to HCs. Postural response patterns were highly variable within groups and seem dependent on the individual. Differences in initial trunk and knee position, enhanced by joint stiffening, could trigger maladaptive postural behavior that predisposes patients to falling. Therefore, rehabilitation programs are important to improve efficient muscle responses and postural awareness, contributing to improved postural stability. In addition, present findings support personalized approaches for balance training in PD.

\section{AUTHOR CONTRIBUTIONS}

$\mathrm{KD}$, AN, and SV: Study design; EB, KD, and SD: Data acquisition. EB, SVR, SV, and AN: Data analysis and interpretation; EB and SVR: Drafting the article; EB, SVR, EH, SV, and AN: Article critical revision for important intellectual content; EB, SVR, EH, KD, SD, SV, and AN: Final approval of the submitted version.

\section{ACKNOWLEDGMENTS}

We are grateful to all the subjects who participated voluntarily in the study. We also would like to thank Professor Friedl De Groote for assistance with data processing and analysis. This study was funded through a grant from Research Foundation Flanders (FWO) [grant number G.0867.15].

\section{SUPPLEMENTARY MATERIAL}

The Supplementary Material for this article can be found online at: https://www.frontiersin.org/articles/10.3389/fneur. 2018.00540/full\#supplementary-material

balance impairment in Parkinson's disease. Mov Disord. (1996) 11:509-21. doi: $10.1002 / \mathrm{mds} .870110506$

5. Carpenter MG, Allum JH, Honegger F, Adkin AL, Bloem BR. Postural abnormalities to multidirectional stance perturbations in Parkinson's disease. J Neurol Neurosurg Psychiatry (2004) 75:1245-54. doi: 10.1136/jnnp.2003.021147

6. Dimitrova D, Horak FB, Nutt JG. Postural muscle responses to multidirectional translations in patients with Parkinson's disease. J Neurophysiol. (2004) 91:489-501. doi: 10.1152/jn.00094.2003

7. Thevathasan W, Pogosyan A, Hyam JA, Jenkinson N, Bogdanovic M, Coyne TJ, et al. A block to pre-prepared movement in gait freezing, 
relieved by pedunculopontine nucleus stimulation. Brain (2011) 134:2085-95. doi: 10.1093/brain/awr131

8. Nonnekes J, de Kam D, Oude Nijhuis LB, van Geel K, Bloem BR, Geurts A, et al. StartReact effects support different pathophysiological mechanisms underlying freezing of gait and postural instability in Parkinson's disease. PLoS ONE (2015) 10:e0122064. doi: 10.1371/journal.pone.0122064

9. Boisgontier MP, Cheval B, Chalavi S, van Ruitenbeek P, Leunissen I, Levin O, et al. Individual differences in brainstem and basal ganglia structure predict postural control and balance loss in young and older adults. Neurobiol Aging (2017) 50:47-59. doi: 10.1016/j.neurobiolaging.2016.10.024

10. Snijders AH, Takakusaki K, Debu B, Lozano AM, Krishna V, Fasano A, et al. Physiology of freezing of gait. Ann Neurol. (2016) 80:644-59. doi: 10.1002/ana.24778

11. Nutt JG, Bloem BR, Giladi N, Hallett M, Horak FB, Nieuwboer A. Freezing of gait: moving forward on a mysterious clinical phenomenon. Lancet Neurol. (2011) 10:734-44. doi: 10.1016/S1474-4422(11)70143-0

12. Duncan RP, Leddy AL, Cavanaugh JT, Dibble LE, Ellis TD, Ford MP, et al. Balance differences in people with Parkinson disease with and without freezing of gait. Gait Posture (2015) 42:306-9. doi: 10.1016/j.gaitpost.2015.06.007

13. Bekkers EMJ, Dijkstra BW, Dockx K, Heremans E, Verschueren SMP, Nieuwboer A. Clinical balance scales indicate worse postural control in people with Parkinson's disease who exhibit freezing of gait compared to those who do not: A meta-analysis. Gait Posture (2017) 56:134-40. doi: 10.1016/j.gaitpost.2017.05.009

14. Smulders K, Esselink RA, De Swart BJ, Geurts AC, Bloem BR, Weerdesteyn V. Postural inflexibility in PD: does it affect compensatory stepping? Gait Posture (2014) 39:700-6. doi: 10.1016/j.gaitpost.2013.10.003

15. Peterson DS, Horak FB. Effects of freezing of gait on postural motor learning in people with Parkinson's disease. Neuroscience (2016) 334:283-9. doi: 10.1016/j.neuroscience.2016.08.017

16. Nieuwboer A, Dom R, De Weerdt W, Desloovere K, Janssens L, Stijn V. Electromyographic profiles of gait prior to onset of freezing episodes in patients with Parkinson's disease. Brain (2004) 127:1650-60. doi: 10.1093/brain/awh189

17. Nieuwboer A, Chavret F, Willems, AM, Desloovere K. Does freezing in Parkinson's disease change limb coordination? J Neurol. (2007) 254:1268-77. doi: 10.1007/s00415-006-0514-3

18. Mohammadi F, Bruijn SM, Vervoort G, van Wegen EE, Kwakkel G, Verschueren S, et al. Motor switching and motor adaptation deficits contribute to freezing of gait in Parkinson's Disease. Neurorehab Neural Repair (2015) 29:132-42. doi: 10.1177/1545968314545175

19. Bekkers EMJ, Hoogkamer W, Bengevoord A, Heremans E, Verschueren SMP, Nieuwboer A. Freezing-related perception deficits of asymmetrical walking in Parkinson's disease. Neuroscience (2017) 364:122-9. doi: 10.1016/j.neuroscience.2017.09.017

20. Manckoundia P, Mourey F, Perennou D, Pfitzenmeyer P. Backward disequilibrium in elderly subjects. Clin Interv Aging (2008) 3:667-72.

21. Hughes AJ, Daniel SE, Kilford L, Lees AJ. Accuracy of clinical diagnosis of idiopathic Parkinson's disease: a clinico-pathological study of 100 cases. $J$ Neurol Neurosurg Psychiatry (1992) 55:181-4.

22. Franchignoni F, Horak F, Godi M, Nardone A, Giordano A. Using psychometric techniques to improve the Balance Evaluation Systems Test: the mini-BESTest. J Rehabil Med. (2010) 42:323-31. doi: 10.2340/165019 77-0537

23. Vanrenterghem J, Gormley D, Robinson M, Lees A. Solutions for representing the whole-body centre of mass in side cutting manoeuvres based on data that is typically available for lower limb kinematics. Gait Posture (2010) 31:517-21. doi: 10.1016/j.gaitpost.2010. 02.014

24. Visser JE, Oude Nijhuis LB, Janssen L, Bastiaanse CM, Borm GF, Duysens J, et al. Dynamic posturography in Parkinson's disease: diagnostic utility of the "first trial effect." Neuroscience (2010) 168:387-94. doi: 10.1016/j.neuroscience.2010.03.068
25. Hof AL, Gazendam MG, Sinke WE. The condition for dynamic stability. J Biomech. (2005) 38:1-8. doi: 10.1016/j.jbiomech.2004. 03.025

26. Bloem BR, van Dijk JG, Beckley DJ, Zwinderman AH, Remler MP, Roos RA. Correction for the influence of background muscle activity on stretch reflex amplitudes. J Neurosci Methods (1993) 46:167-74.

27. McKay JL, Ting LH, Hackney ME. Balance, body motion, and muscle activity after high-volume short-term dance-based rehabilitation in persons with Parkinson Disease: a pilot study. J Neurol Phys Ther. (2016) 40:257-68. doi: 10.1097/NPT.0000000000000150

28. Falconer K, Winter DA. Quantitative assessment of co-contraction at the ankle joint in walking. Electromyogr Clin Neurophysiol. (1985) 25:135-49.

29. Horak FB, Dimitrova D, Nutt JG. Direction-specific postural instability in subjects with Parkinson's disease. Exp Neurol. (2005) 193:504-21. doi: 10.1016/j.expneurol.2004.12.008

30. Schlenstedt C, Muthuraman M, Witt K, Weisser B, Fasano A, Deuschl G. Postural control and freezing of gait in Parkinson's disease. Parkinsonism Relat Disord. (2016) 24:107-12. doi: 10.1016/j.parkreldis.2015. 12.011

31. Jacobs JV, Dimitrova DM, Nutt JG, Horak FB. Can stooped posture explain multidirectional postural instability in patients with Parkinson's disease? Exp Brain Res. (2005) 166:78-88. doi: 10.1007/s00221-0052346-2

32. Takakusaki K, Saitoh K, Harada H, Kashiwayanagi M. Role of basal gangliabrainstem pathways in the control of motor behaviors. Neurosci Res. (2004) 50:137-51. doi: 10.1016/j.neures.2004.06.015

33. Takakusaki K. Functional neuroanatomy for posture and gait control. J Mov Disord. (2017) 10:1-17. doi: 10.14802/jmd.16062

34. Stapley PJ, Drew T. The pontomedullary reticular formation contributes to the compensatory postural responses observed following removal of the support surface in the standing cat. J Neurophysiol. (2009) 101:1334-50. doi: $10.1152 /$ jn. 91013.2008

35. Lu C, Amundsen Huffmaster SL, Harvey JC, MacKinnon CD. Anticipatory postural adjustment patterns during gait initiation across the adult lifespan. Gait Posture (2017) 57:182-7. doi: 10.1016/j.gaitpost.2017.06.010

36. Allen JL, McKay JL, Sawers A, Hackney ME, Ting LH. Increased neuromuscular consistency in gait and balance after partnered, dancebased rehabilitation in Parkinson's disease. J Neurophysiol (2017) 118:363-73. doi: $10.1152 /$ jn. 00813.2016

37. Vandenbossche J, Deroost N, Soetens E, Coomans D, Spildooren J, Vercruysse $S$, et al. Impaired implicit sequence learning in Parkinson's disease patients with freezing of gait. Neuropsychology (2013) 27:28-36. doi: 10.1037/a0031278

38. Horak FB, Frank J, Nutt J. Effects of dopamine on postural control in Parkinsonian subjects: scaling, set, and tone. J Neurophysiol. (1996) 75:2380-96.

39. Bonnet CT, Delval A, Szaffarczyk S, Defebvre L. Levodopa has primarily negative influences on postural control in patients with Parkinson's disease. Behav Brain Res. (2017) 331:67-75. doi: 10.1016/j.bbr.2017.05.033

40. Curtze C, Nutt JG, Carlson-Kuhta P, Mancini M, Horak FB. Levodopa is a double-edged sword for balance and gait in people with Parkinson's Disease. Mov Disord. (2015) 30:1361-70. doi: 10.1002/mds. 26269

Conflict of Interest Statement: The authors declare that the research was conducted in the absence of any commercial or financial relationships that could be construed as a potential conflict of interest.

Copyright $(92018$ Bekkers, Van Rossom, Heremans, Dockx, Devan, Verschueren and Nieuwboer. This is an open-access article distributed under the terms of the Creative Commons Attribution License (CC BY). The use, distribution or reproduction in other forums is permitted, provided the original author(s) and the copyright owner(s) are credited and that the original publication in this journal is cited, in accordance with accepted academic practice. No use, distribution or reproduction is permitted which does not comply with these terms. 